\title{
Combination of laparoscopic and endoscopic approaches for neoplasia with non-exposure technique (CLEAN-NET) for gastric submucosal tumors: updated advantages and limitations
}

\author{
Manabu Onimaru, Haruhiro Inoue, Haruo Ikeda, Mary Raina Angeli Abad, Bianca Maria Quarta Colosso, \\ Yuto Shimamura, Kazuya Sumi, Yoshio Deguchi, Hiroaki Ito, Noboru Yokoyama \\ Digestive Diseases Center, Showa University Koto Toyosu Hospital, Tokyo, Japan \\ Contributions: (I) Conception and design: M Onimaru, H Inoue; (II) Administrative support: Y Deguchi, H Ito, N Yokoyama; (III) Provision of \\ study materials or patients: M Onimaru, H Ikeda, MRA Abad, BM Quarta Colosso, Y Shimamura, K Sumi; (IV) Collection and assembly of data: \\ M Onimaru, H Ikeda, MRA Abad, BM Quarta Colosso, Y Shimamura, K Sumi; (V) Data analysis and interpretation: M Onimaru; (VI) Manuscript \\ writing: All authors; (VII) Final approval of manuscript: All authors. \\ Correspondence to: Manabu Onimaru, MD, PhD. Digestive Diseases Center, Showa University Koto Toyosu Hospital, 5-1-38, Toyosu, Koto-ku, \\ Tokyo, 135-8577, Japan. Email: mnbonmr0827@gmail.com.
}

\begin{abstract}
Laparoscopic and endoscopic cooperative surgery (LECS) for gastric submucosal tumors (SMTs) has been developed under the concept of resecting gastric tumors with both complete curability and preserving organ functions. Precise resection is obtained by classical LECS, however, concerns regarding intraoperative bacterial infection and dissemination of the tumor cells into the abdominal cavity by LECS with exposure technique still remain. To prevent these concerns, several LECS-related procedures with nonexposure techniques, such as combination of laparoscopic and endoscopic approaches for neoplasia with nonexposure technique (CLEAN-NET) and non-exposed endoscopic wall-inversion surgery (NEWS), have been reported to be safe and feasible. Classical LECS, CLEAN-NET, and NEWS have the same concept, however, each has its own different characteristic procedures; exposure or non-exposure technique, inversion of the tumor into or outer the lumen, retrieval of tumor per oral or through the abdominal cavity, and dominance in the role of the endoscopist or the laparoscopic surgeon. Familiarization with these procedure details is important to understand their indications, advantages and limitations, resulting in providing a tailored minimally invasive surgery for patients. The main scope of this review article is to introduce readers to the clinical application, procedure, and results of CLEAN-NET, both from previous literatures and from our experience, as well as to offer a closer look at its advantages and limitations while comparing with other LECS procedures from the viewpoint of introducing CLEAN-NET first.
\end{abstract}

Keywords: Laparoscopic and endoscopic cooperative surgery (LECS); combination of laparoscopic and endoscopic approaches for neoplasia with non-exposure technique (CLEAN-NET); non-exposed endoscopic wallinversion surgery (NEWS); gastric submucosal tumors (SMTs)

Submitted Jul 17, 2019. Accepted for publication Sep 02, 2019.

doi: 10.21037/atm.2019.09.19

View this article at: http://dx.doi.org/10.21037/atm.2019.09.19 


\section{Introduction}

Complete surgical resection is currently considered the first choice of treatment for patients with resectable gastric submucosal tumors (SMTs) including gastrointestinal stromal tumors (GISTs) (1-3). For complete surgical en bloc resection of gastric SMTs, an adequate safety margin should be secured without injuring the pseudocapsule of the SMTs during resection (1). Laparoscopic gastric surgery has been advanced and standardized worldwide. Furthermore, laparoscopic wedge resection for gastric SMTs has also been reported to be safe, oncologically feasible, and with a decrease in the length of hospital stay and blood loss (4-6). For SMTs with extraluminal growth, laparoscopic wedge resection is easier to detect, however, when they grow in the intra-lumen, identification and determination of appropriate free margin of the SMT from the outside of the stomach is difficult, sometimes resulting in postoperative gastroparesis due to excessive resection, deformity or stenosis of the stomach.

In order to overcome these concerns, Hiki et al. first reported laparoscopic and endoscopic cooperative surgery (LECS), currently named classical LECS, for gastric GISTs in 2008 (7). The procedure of classical LECS is as follows: (I) determination of accurate incision line from the endoscopic view; (II) endoscopic submucosal resection around the tumor; (III) artificial perforation of the stomach by endoscopic device along the resection line; (IV) seromuscular dissection by endoscopic or laparoscopic device, $(\mathrm{V})$ removal of the tumor from the abdominal cavity; and (VI) closure of the opened gastric wall with handsewn sutures or laparoscopic stapling device (Figure 1A). This technique produces an appropriate margin, which is keeping an oncological enough distance from the tumor with minimizing resected stomach. Classical LECS has been reported to be safe and with excellent results (7-13), however, there have been concerns regarding the risks of scattering and seeding of bacterial contamination or tumor cells into the abdominal cavity due to the perforation of the gastric wall.

To avoid these concerns, several modified LECS techniques without exposure of the gastric mucosa were reported. Combination of laparoscopic and endoscopic approaches for neoplasia with non-exposure technique (CLEAN-NET) (14) and Non-exposed endoscopic wallinversion surgery (NEWS) (15-17) were among the representatives of non-exposure techniques (Figure 1B,C). Since we developed the first CLEAN-NET in 2012 (14), only a few reports regarding the results of CLEAN-NET have been published so far (18-21).

The main scope of this review article is to introduce readers to the clinical application, procedure, and results of the CLEAN-NET, both from previous literatures and from our experience, as well as to offer a closer look at its advantages and limitations while comparing with other LECS procedures, classical LECS, and NEWS.

\section{Procedures of CLEAN-NET}

CLEAN-NET is a technique of non-exposure full-thickness gastric wall resection performed under the combination of laparoscopic and endoscopic approaches. The procedure of CLEAN-NET for SMTs is as follows: (I) endoscopic marking around the tumor from the intra-lumen of the stomach; (II) laparoscopic marking around the tumor on the serosa under the assistance of the endoscopic confirmation of the resection line; (III) laparoscopic seromuscular dissection around the tumor along to the resection line; (IV) lifting the tumor toward the abdominal cavity for obtaining definite margin-free resection; (V) full-layer resection with a laparoscopic linear stapling device; (VI) transabdominal retrieval of the specimen wrapped with a collecting bag; and (VII) checking by intraoperative endoscopy after resection (14) [Figure 1C (upper); Figure 2]. Fujishima $e t a l$. and Kanehira et al. reported a further modified CLEAN-NET technique, altering the final cutand-closure procedure from stapling full-layer cut-andclosure to stapling only the mucosal layer and adding seromuscular closure by hand-sewn suturing $(19,21)$ [Figure 1C (below)].

\section{Outcomes of CLEAN-NET}

Published data of the outcomes of the original/modified CLEAN-NET for gastric SMTs are shown in Table 1. Previous reports on CLEAN-NET are relatively few, with only a small number of patients included. The total number of patients was 75 . The average tumor size was approximately $35 \mathrm{~mm}$. The maximum tumor size was $90 \mathrm{~mm}$, since Kanehira et al. included patients who were selected for modified CLEAN-NET even when the tumor was larger than $5 \mathrm{~cm}$, or when the base of the tumor included in the gastric wall was less than $5 \mathrm{~cm}$ and the tumor was exophytic. The location of the tumors was widely distributed in the stomach including lesions adjacent to esophagogastric junction (EGJ) or the pyloric ring, however, in a report of the largest series, SMTs located adjacent 


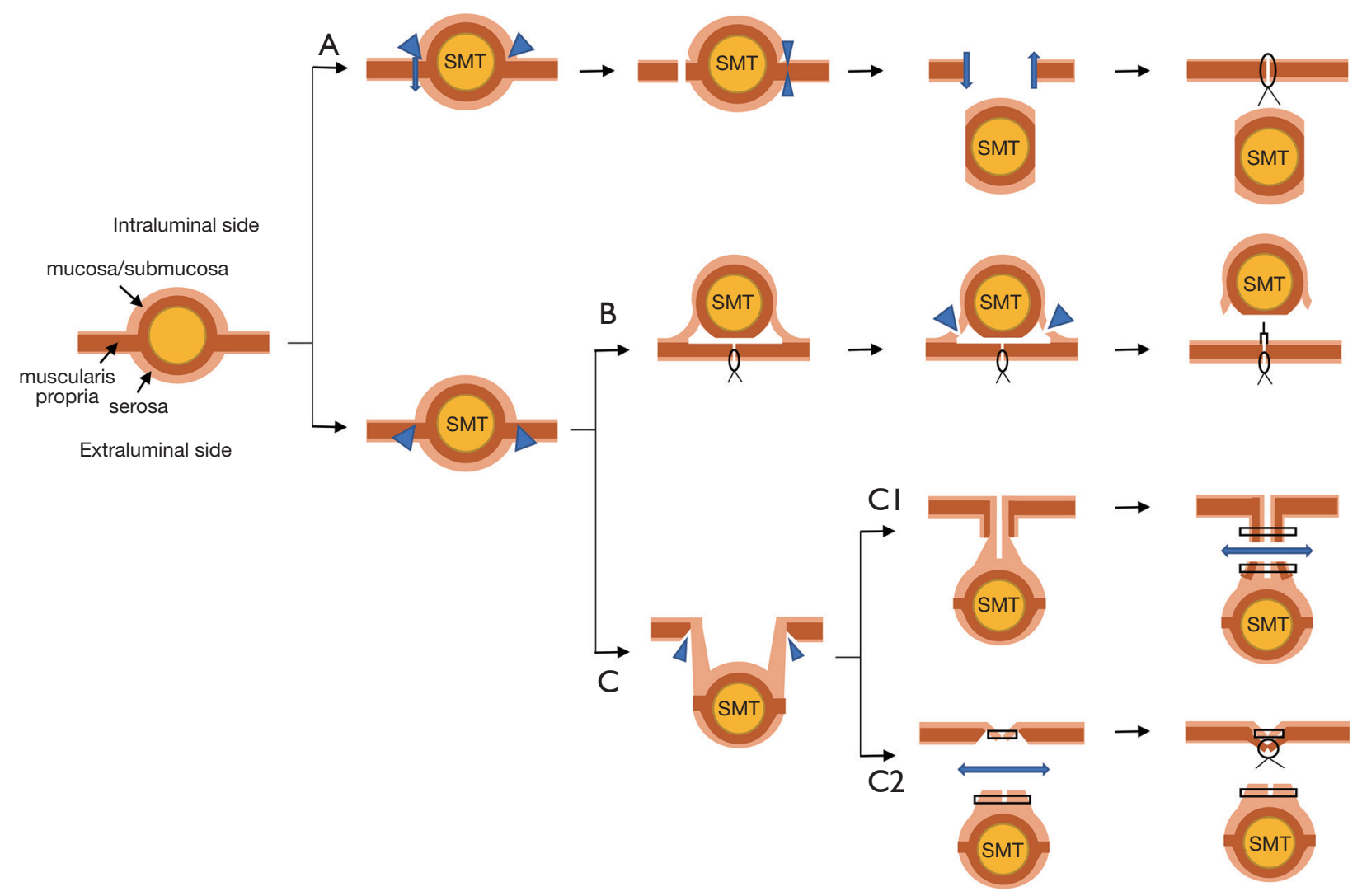

Figure 1 Schema of the procedures: (A) classical LECS (B) NEWS (C1) CLEAN-NET and (C2) modified CLEAN-NET. LECS, laparoscopic and endoscopic cooperative surgery; NEWS, non-exposed endoscopic wall-inversion surgery; CLEAN-NET, combination of laparoscopic and endoscopic approaches for neoplasia with non-exposure technique.

to EGJ were excluded from the indicated criteria (21). The operation time, blood loss, and the length of hospitalization were approximately 120 minutes, $7-8 \mathrm{~g}$, and 6.0 days, respectively. Only 1 case $(1.3 \%)$, wherein the tumor was located on the posterior wall of the middle stomach, was reported to have a postoperative complication of stenosis (19). Importantly, 50 cases out of a total of 75 cases were GISTs, and all were completely resected and were reported without any recurrences.

Our clinical results of the original CLEAN-NET for gastric SMTs were also shown in Figure 3 and Table 2. These 36 cases included 8 cases from our first published report (14), and had almost the same results as the published results so far. In one patient, postoperative gastroparesis, which needed reoperation (distal gastrectomy), was observed. In this case, the tumor was located on the posterior gastric angle with a $40 \mathrm{~mm}$ size and resected with a minimum tumor-free margin by the original CLEAN-NET. Intraoperative endoscopic examination confirmed that the staple line was distant enough from the pyloric ring and the remnant stomach had a minimal deformity without difficulty in passing the endoscope through the pyloric ring. However, two months after CLEAN-NET, the patient had symptoms of gastroparesis. Postoperative endoscopic examination revealed a delay in the shortening of the distance from the staple line to the pyloric ring caused the poor outflow passage of the stomach (Figure 4).

\section{Discussion}

The concept of LECS is to resect gastric tumors with both complete curability and preserving organ functions. Similar to classical LECS and NEWS, previous literatures and our series revealed that CLEAN-NET is also a safe and feasible technique while maintaining the concept of LECS. These techniques have the same concept, however, each technique has different and unique manipulations; exposure or nonexposure technique, inversion of the tumor into or outer the 

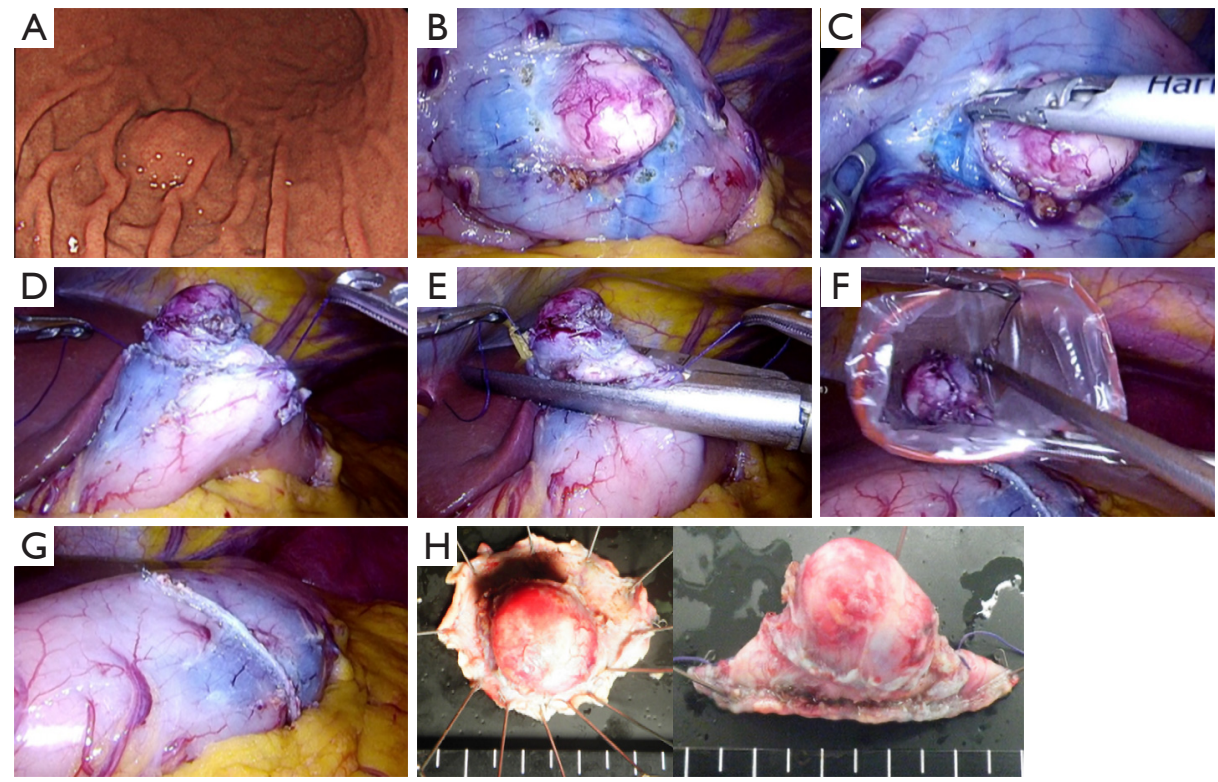

Figure 2 Procedure of CLEAN-NET: (A) preoperative endoscopy showed a $30 \mathrm{~mm}$ SMT with intraluminal growth at the greater curvature in the middle stomach; (B) laparoscopic marking around the tumor on the serosa under the assistance of the endoscopic confirmation of the resection line; (C) laparoscopic seromuscular dissection around the tumor along the resection line; (D) lifting the tumor toward the abdominal cavity for obtaining definite margin-free resection; (E) full-layer resection with a laparoscopic linear stapling device; (F) transabdominal retrieval of the specimen wrapped with a collecting bag; $(\mathrm{G})$ checking by intraoperative endoscopy after resection; $(\mathrm{H})$ resected specimen diagnosed as schwannoma having a $23 \mathrm{~mm} \times 20 \mathrm{~mm} \times 18 \mathrm{~mm}$ size with free-margin from the tumor. CLEAN-NET, combination of laparoscopic and endoscopic approaches for neoplasia with non-exposure technique; SMT, submucosal tumor.

lumen, retrieval of tumor per oral or through the abdominal cavity, and dominance in the role of the endoscopist or the laparoscopic surgeon.

We herein review and discuss the advantages and limitations of CLEAN-NET in comparison with the characteristics of classical LECS and NEWS.

\section{Patient characteristics}

In these series, CLEAN-NET was performed safely on patients over 80 years old or with comorbidities. The operation time, blood loss, and length of hospitalization were acceptable, and no operation-related systemic adverse events occurred. Therefore, it has been concluded that CLEAN-NET is also a minimally invasive and safe procedure similar to classical LECS and NEWS.

\section{Tumor size}

Laparoscopic resection of gastric GISTs smaller than $5 \mathrm{~cm}$ was reported to be safe when performed by experts (22-24).
In most of these reports, a tumor size of $>5 \mathrm{~cm}$ was excluded from the indication criteria. Considering the tumor was retrieved per orally, tumor size was limited to $<3 \mathrm{~cm}$ in NEWS. Meanwhile, since the tumor was retrieved transabdominally in CLEAN-NET, there was no limitation in tumor size. Interestingly, Kanehira et al. resected a tumor with $90 \mathrm{~mm}$ size and reported that a tumor size of $>5 \mathrm{~cm}$ is possible to be resected safely if the tumor base included in the gastric wall is $<5 \mathrm{~cm}$ (21). However, when the size of the resected specimen is $>5 \mathrm{~cm}$, even though CLEAN-NET is completed safely, deformity of the remnant stomach may highly occur, leading to postoperative stenosis or gastroparesis. As shown in Figure 5, this case was excluded from the indication of CLEAN-NET due to its tumor size and performing of distal gastrectomy (Figure 5). Furthermore, from the oncological view, NCCN guidelines and Japanese guidelines state that laparoscopic resection of GISTs $>5 \mathrm{~cm}$ or GISTs $<5 \mathrm{~cm}$ with malignant character is not recommended $(2,25,26)$. Therefore, CLEAN-NET can be applied technically for any size of gastric SMTs, however, CLEAN-NET for SMTs $>5 \mathrm{~cm}$ should be carefully applied 


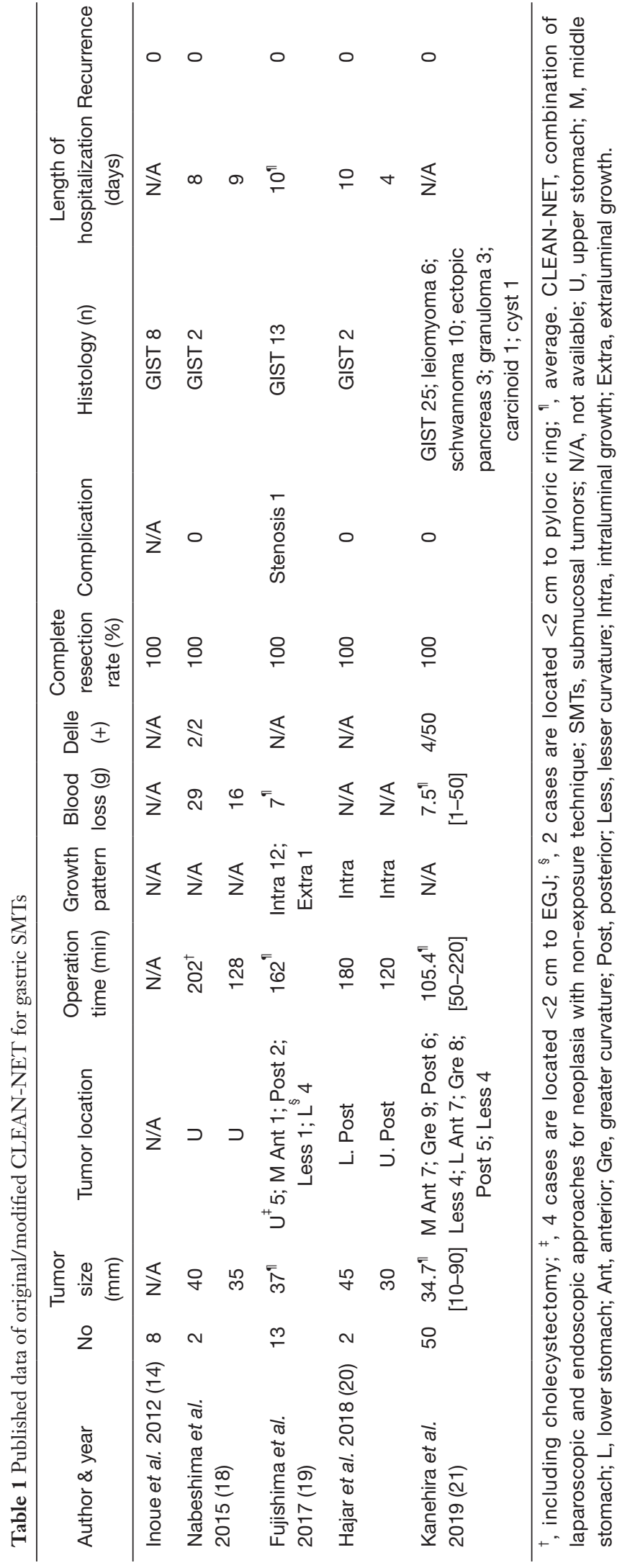

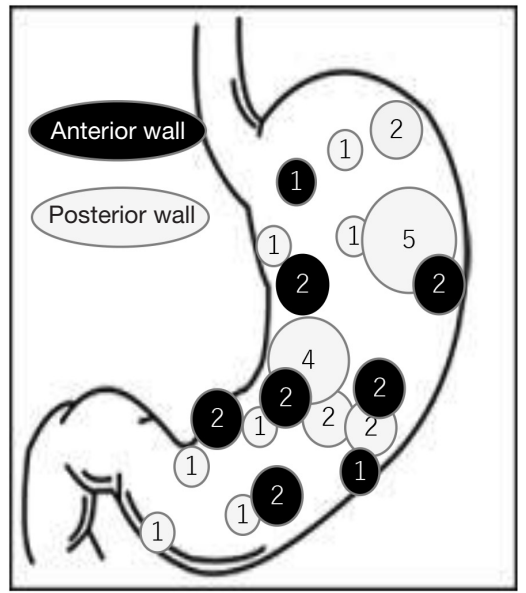

Figure 3 Location of the SMTs performed CLEAN-NET: SMTs were distributed in any lesions of the stomach. Black circle, white circle, and numerals in the circle represented locations on the anterior wall, locations on the posterior wall, and numbers of patients, respectively. CLEAN-NET, combination of laparoscopic and endoscopic approaches for neoplasia with non-exposure technique; SMT, submucosal tumor.

in the view of postoperative dysfunction and oncology.

\section{Location of the tumor}

Gastric SMTs located adjacent to EGJ and the pyloric ring, or on the lesser curvature are sometimes technically demanding since it is easier to result in postoperative stenosis in these locations. Classical LECS was reported to be applied for SMTs in these lesions since classical LECS can obtain precise control of the incision line from the inside of the stomach (10). On the other hand, CLEANNET for SMTs within a $1 \mathrm{~cm}$ distance from the EGJ or the pyloric ring might be challenging, since closing the incised seromuscular line by laparoscopic linear stapling device has the potential of excessive loss of the stomach. As mentioned above in the Procedures of CLEAN-NET, Fujishima and Kanehira modified the original CLEANNET. Fujishima et al. reported that this modified CLEANNET, termed serosal and muscular layers incision technique (SAMIT), could resect 6 cases of the gastric SMTs within $2 \mathrm{~cm}$ distance from the EGJ or the pyloric ring without any stenosis or stasis (19). By using this modified technique, CLEAN-NET might be applied for SMTs in any lesions. Moreover, in lesions found on the lesser curvature, cutand-close by using a linear stapling device may sometimes 
Table 2 Patients' demographics and clinical results of CLEAN-NET for gastric SMTs $(\mathrm{N}=36)$

\begin{tabular}{ll}
\hline Variables & Results \\
\hline Gender (male/female) & $21 / 15$ \\
Age (average, min-max) & 59.9 [25-87] \\
ASA physical status classification & $15 / 21 / 0 / 0 / 0 / 0$ \\
(I/II/III/IV/VI) & \\
Growth pattern & $33 / 3$ \\
(intraluminal/extraluminal) & \\
Tumor size (average, min-max) & $31.3[15-63]$ mm \\
Dell (+/-) & $9 / 27$ \\
Tumor location & Shown in Figure 3 \\
Operation time (average, min-max) & 112.7 (30-198) min \\
Blood loss (average, min-max) & $8.7(0-135) \mathrm{g}$ \\
Margin negative & $36(100 \%)$ \\
Pathological diagnosis (n) & GIST: 27; schwannoma: \\
& $5 ;$ aberrant pancreas: 2; \\
leiomyoma: $1 ;$ \\
hollow-up period (days) & hamartoma: 1 \\
\hline Rectoperative complications & $1(2.8 \%)$ gastroparesis \\
& 0 (0\%) \\
& 1,387 [33-3,294] \\
\hline
\end{tabular}

CLEAN-NET, combination of laparoscopic and endoscopic approaches for neoplasia with non-exposure technique; SMTs, submucosal tumors.

cause severe deformity or damage to the Latarjet branch of the vagal nerve, leading to a delay in gastric emptying. Therefore, when a large-sized tumor is located on the lesser curvature, minimal area of blood vessel manipulation and closure by hand-sewn suturing might solve these concerns.

Tumor location also directly affects the accessibility to the tumor. When the tumor is located on the posterior wall, the laparoscopic approach is somewhat more challenging. Similarly, when the tumor is located in the fornix, procedures which include endoscopic submucosal resection such as classical LECS and NEWS may sometimes be technically demanding.

\section{Delle/ulceration}

It is still controversial whether there are risks of bacterial infection and tumor dissemination when gastric content is exposed into the abdominal cavity during manipulation. Mori et al. reported the possibility of bacterial infection and reduction effect by preoperative saline lavage of the stomach (27), in comparison to a report by Ikehara et al. stating that perforation associated with EMR and ESD did not lead to peritoneal dissemination (28). As of present, SMTs with delle/ulceration are considered better to be resected with non-exposure technique.

\section{Growth pattern}

When the tumor grows extraluminally, the tumor base on the gastric wall is usually small. In these lesions, laparoscopic simple wedge resection with the linear stapling device has minimal risks of postoperative deformity, and it is easier to perform hence shortening the time of the procedure. Therefore, LECS and LECS-related techniques are not necessary to be applied to SMTs with extraluminal growth. On the contrary, these should be applied to the SMTs with intraluminal growth.

\section{Cost}

Both endoscopic dissecting devices and laparoscopic devices are needed for Classical LECS and NEWS, while CLEANNET can be completed only by using laparoscopic devices. However, linear stapling devices, which are cost-consuming, must be used for the cut-and-closing procedure in CLEANNET, while classical LECS and NEWS can be closed by hand-sewn suturing. When the situations of the tumor are allowed, reducing cost might be a benefit for patients and should be considered.

\section{Technical issue}

CLEAN-NET was developed based on the concept that minimizing the resected seromuscular area, not mucosal area, of the stomach may contribute to the minimization of deformity. This concept was the reason why most of the approaches were done from the outside of the stomach (seromuscular dissection, cutting, and closing), and the process of lifting-up the lesion, which allows minimum resection of the seromuscular area with a wide mucosal tumor-free margin, reflects this concept.

The procedure of cut-and-closing by linear stapling device in CLEAN-NET results easier and reduced procedure time compared to other techniques. In addition, other LECS and LECS-related procedures require endoscopists to be familiar with the endoscopic treatment 

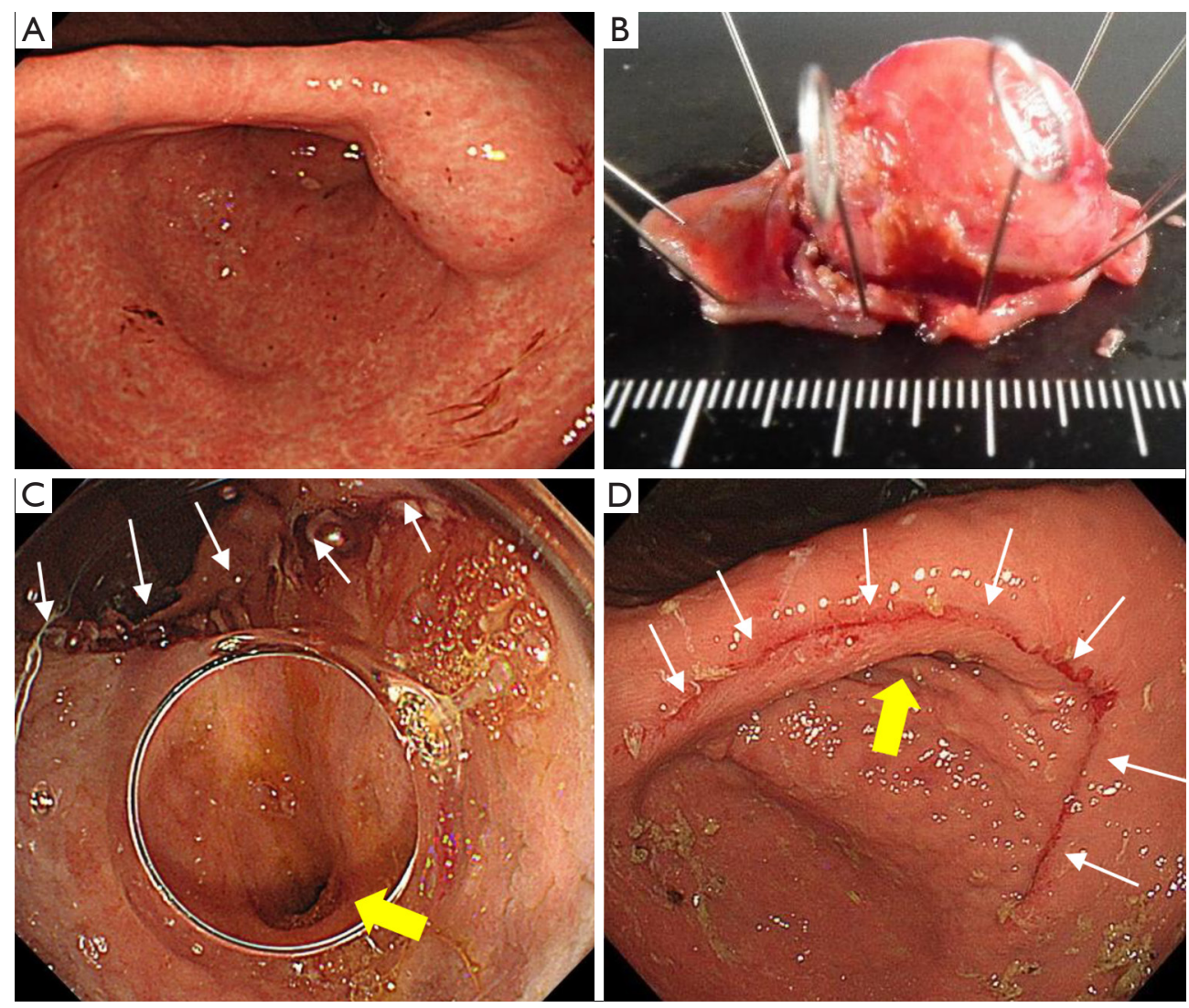

Figure 4 A case of postoperative gastroparesis after CLEAN-NET: (A) A $40 \mathrm{~mm} \times 40 \mathrm{~mm}$ size intraluminal growth GIST was located on the posterior gastric angle; (B) GIST was resected with a minimum tumor-free margin by CLEAN-NET; (C) intraoperative endoscopy confirmed that the staple line (white arrow) was distant enough from the pyloric ring (yellow arrow) and remnant stomach had minimal deformity; (D) endoscopic examination after 2 months revealed a delayed shortening of the distance from staple line (white arrow) to the pyloric ring (yellow arrow), leading to postoperative gastroparesis. CLEAN-NET, combination of laparoscopic and endoscopic approaches for neoplasia with nonexposure technique; GIST, gastrointestinal stromal tumor.
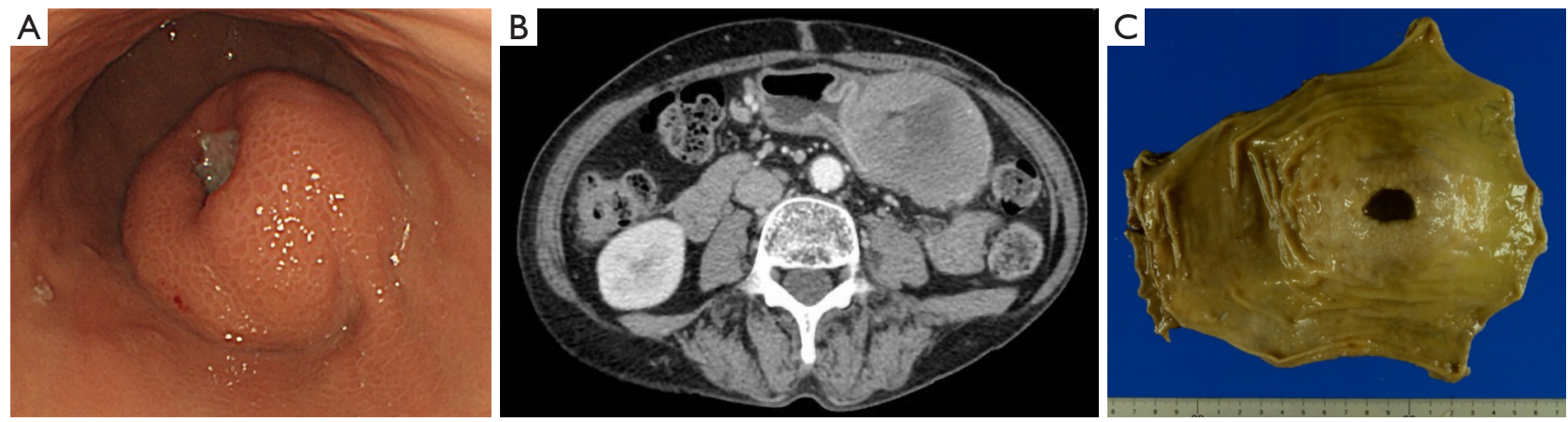

Figure 5 A case of SMT excluded from the indication criteria of CLEAN-NET: (A) Endoscopic view showed a large 70 mm SMT, located at the lower stomach with delle; (B) CT scan also showed a large $70 \mathrm{~mm}$ SMT with intraluminal growth; (C) distal gastrectomy was selected to avoid strong deformity after CLEAN-NET. Pathological diagnosis was schwannoma. SMT, submucosal tumor; CLEAN-NET, combination of laparoscopic and endoscopic approaches for neoplasia with non-exposure technique. 
Table 3 Characteristics of laparoscopic simple wedge resection, classical LECS, NEWS and CLEAN-NET

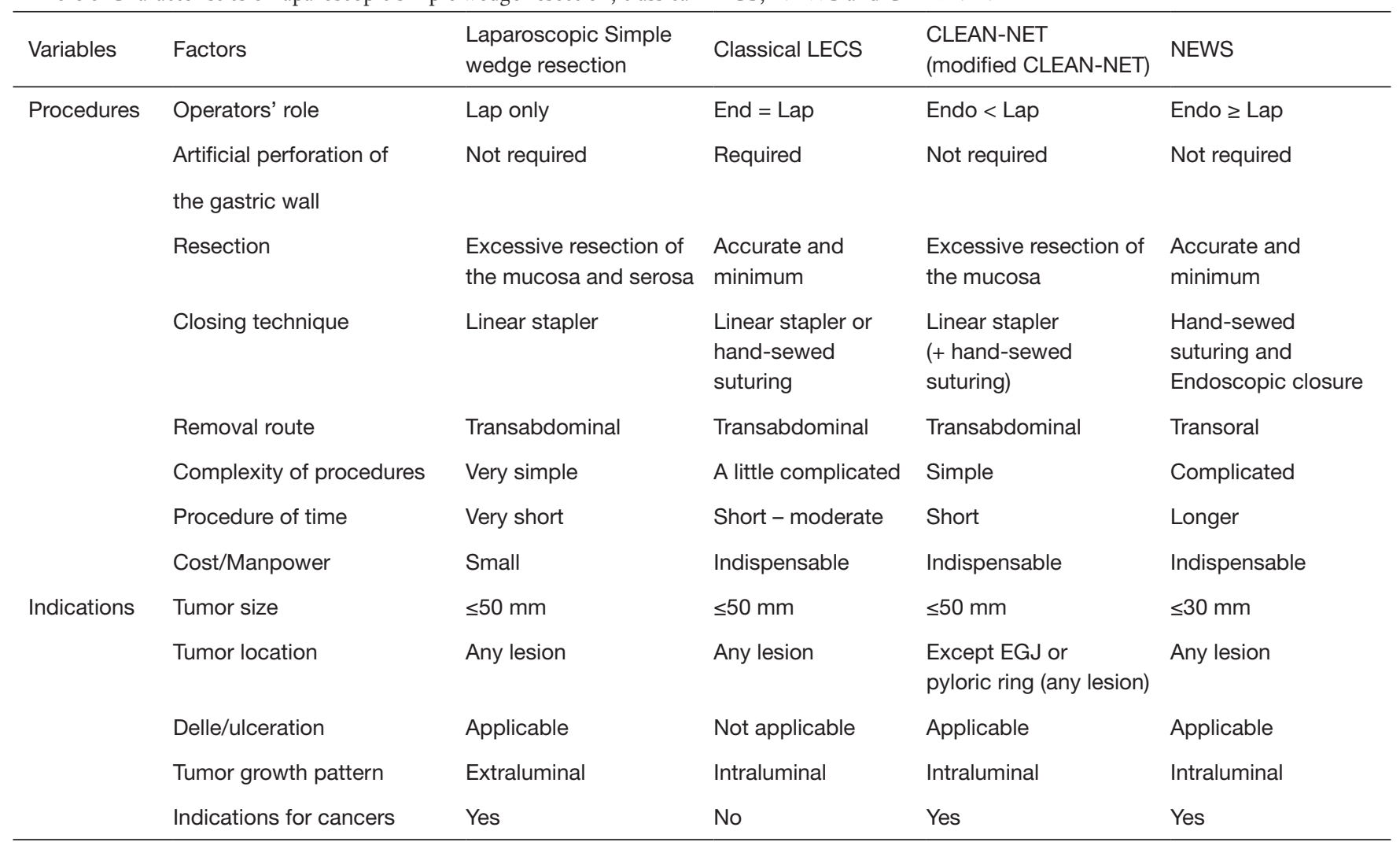

Lap, laparoscopists; End, endoscopists; LECS, laparoscopic and endoscopic cooperative surgery; NEWS, non-exposed endoscopic wall-inversion surgery; CLEAN-NET, combination of laparoscopic and endoscopic approaches for neoplasia with non-exposure technique.

including submucosal resection (ESD), therefore, CLEANNET may be easier for the introduction and standardization of LECS procedures.

Summary of characteristics of classical LECS, NEWS, and CLEAN-NET were shown in Table 3.

\section{A further direction of CLEAN-NET}

For gastric cancers, regardless of the improvement of the ESD technique, there still remains lesions which are technically difficult to complete ESD due to the tumor locations or non-lifting lesions with severe scars. When the lesions meet the inclusion criteria of ESD, LECS with non-exposure technique is applied in some limited institutions. Inoue et al. (14) and Goto et al. (29) reported the results and possibility of applying CLEAN-NET and NEWS for early gastric cancers, respectively. In the recent years, favorable results of minimally invasive function-preserving gastrectomy with sentinel lymph node navigation surgery have been reported (30-34). Perhaps in the near future, sentinel lymph node navigation surgery may be established, and a tailored minimally invasive surgery can be provided.

\section{Conclusions}

CLEAN-NET is one of the safe and feasible LECS-related procedures with a non-exposure technique for gastric SMTs. In selecting a procedure, familiarization with the characteristics (indications, procedural pitfalls, advantages and limitations) of each procedure is important to secure the curability and postoperative function, and it may provide further applications of LECS procedures.

\section{Acknowledgments}

We thank Prof. Shin-ei Kudo and Prof. Fumio Ishida at Digestive Diseases Center, Showa University Northern Yokohama Hospital, Yokohama, Japan, for the kind contribution in data collection. We are also grateful to all 
the patients and clinical staff.

\section{Footnote}

Conflicts of Interest: $\mathrm{H}$ Inoue is an advisor of Olympus Corporation and Top Corporation. He has also received educational grants from Olympus Corp., and Takeda Pharmaceutical Co. The other authors have no conflicts of interest to declare.

Ethical Statement: The authors are accountable for all aspects of the work in ensuring that questions related to the accuracy or integrity of any part of the work are appropriately investigated and resolved.

\section{References}

1. Koo DH, Ryu MH, Kim KM, et al. Asian Consensus Guidelines for the Diagnosis and Management of Gastrointestinal Stromal Tumor. Cancer Res Treat 2016;48:1155-66.

2. Nishida T, Hirota S, Yanagisawa A, et al. Clinical practice guidelines for gastrointestinal stromal tumor (GIST) in Japan: English version. Int J Clin Oncol 2008;13:416-30.

3. Iwahashi M, Takifuji K, Ojima T, et al. Surgical management of small gastrointestinal stromal tumors of the stomach. World J Surg 2006;30:28-35.

4. Choi SM, Kim MC, Jung GJ, et al. Laparoscopic wedge resection for gastric GIST: long-term follow-up results. Eur J Surg Oncol 2007;33:444-7.

5. Matsuhashi N, Osada S, Yamaguchi K, et al. Long-term outcomes of treatment of gastric gastrointestinal stromal tumor by laparoscopic surgery: review of the literature and our experience. Hepatogastroenterology 2013;60:2011-5.

6. Melstrom LG, Phillips JD, Bentrem DJ, et al. Laparoscopic versus open resection of gastric gastrointestinal stromal tumors. Am J Clin Oncol 2012;35:451-4.

7. Hiki N, Yamamoto Y, Fukunaga T, et al. Laparoscopic and endoscopic cooperative surgery for gastrointestinal stromal tumor dissection. Surg Endosc 2008;22:1729-35.

8. Hiki N, Nunobe S, Matsuda T, et al. Laparoscopic endoscopic cooperative surgery. Dig Endosc 2015;27:197-204.

9. Tsujimoto H, Yaguchi Y, Kumano I, et al. Successful gastric submucosal tumor resection using laparoscopic and endoscopic cooperative surgery. World J Surg 2012;36:327-30.

10. Hoteya S, Haruta S, Shinohara H, et al. Feasibility and safety of laparoscopic and endoscopic cooperative surgery for gastric submucosal tumors, including esophagogastric junction tumors. Dig Endosc 2014;26:538-44.

11. Matsuda T, Hiki N, Nunobe $S$, et al. Feasibility of laparoscopic and endoscopic cooperative surgery for gastric submucosal tumors (with video). Gastrointest Endosc 2016;84:47-52.

12. Matsuda T, Nunobe S, Kosuga T, et al. Laparoscopic and luminal endoscopic cooperative surgery can be a standard treatment for submucosal tumors of the stomach: a retrospective multicenter study. Endoscopy 2017;49:476-83.

13. Kang WM, Yu JC, Ma ZQ, et al. Laparoscopic-endoscopic cooperative surgery for gastric submucosal tumors. World J Gastroenterol 2013;19:5720-6.

14. Inoue H, Ikeda H, Hosoya T, et al. Endoscopic mucosal resection, endoscopic submucosal dissection, and beyond: full-layer resection for gastric cancer with nonexposure technique (CLEAN-NET). Surg Oncol Clin N Am 2012;21:129-40.

15. Mitsui T, Niimi K, Yamashita H, et al. Non-exposed endoscopic wall-inversion surgery as a novel partial gastrectomy technique. Gastric Cancer 2014;17:594-9.

16. Goto O, Takeuchi H, Sasaki M, et al. Laparoscopy-assisted endoscopic full-thickness resection of gastric subepithelial tumors using a nonexposure technique. Endoscopy 2016;48:1010-5.

17. Mitsui T, Yamashita H, Aikou S, et al. Non-exposed endoscopic wall-inversion surgery for gastrointestinal stromal tumor. Transl Gastroenterol Hepatol 2018;3:17.

18. Nabeshima K, Tomioku M, Nakamura K, et al. Combination of Laparoscopic and Endoscopic Approaches to Neoplasia with Non-exposure Technique (CLEANNET) for GIST with Ulceration. Tokai J Exp Clin Med 2015;40:115-9.

19. Fujishima H, Etoh T, Hiratsuka T, et al. Serosal and muscular layers incision technique in laparoscopic surgery for gastric gastrointestinal stromal tumors. Asian J Endosc Surg 2017;10:92-5.

20. Hajer J, Havluj L, Whitley A, et al. Non-Exposure Endoscopic-Laparoscopic Cooperative Surgery for Stomach Tumors: First Experience from the Czech Republic. Clin Endosc 2018;51:167-73.

21. Kanehira E, Kanehira AK, Tanida T, et al. CLEANNET: a modified laparoendoscopic wedge resection of the stomach to minimize the sacrifice of innocent gastric wall. Surg Endosc 2019. [Epub ahead of print].

22. Otani Y, Furukawa T, Yoshida $M$, et al. Operative indications for relatively small $(2-5 \mathrm{~cm})$ gastrointestinal 
stromal tumor of the stomach based on analysis of 60 operated cases. Surgery 2006;139:484-92 .

23. Novitsky YW, Kercher KW, Sing RF, et al. Longterm outcomes of laparoscopic resection of gastric gastrointestinal stromal tumors. Ann Surg 2006;243:73845; discussion 745-7.

24. Nishimura J, Nakajima K, Omori T, et al. Surgical strategy for gastric gastrointestinal stromal tumors: laparoscopic vs. open resection. Surg Endosc 2007;21:875-8.

25. Nishida T, Blay JY, Hirota S, et al. The standard diagnosis, treatment, and follow-up of gastrointestinal stromal tumors based on guidelines. Gastric Cancer 2016;19:3-14.

26. Demetri GD, von Mehren M, Antonescu CR, et al. NCCN Task Force report: update on the management of patients with gastrointestinal stromal tumors. J Natl Compr Canc Netw 2010;8 Suppl 2:S1-41; quiz S42-4.

27. Mori H, Kobara H, Tsushimi T, et al. Reduction effect of bacterial counts by preoperative saline lavage of the stomach in performing laparoscopic and endoscopic cooperative surgery. World J Gastroenterol 2014;20:15763-70.

28. Ikehara H, Gotoda T, Ono H, et al. Gastric perforation during endoscopic resection for gastric carcinoma and the

Cite this article as: Onimaru $\mathrm{M}$, Inoue $\mathrm{H}$, Ikeda $\mathrm{H}$, Abad MRA, Quarta Colosso BM, Shimamura Y, Sumi K, Deguchi Y, Ito H, Yokoyama N. Combination of laparoscopic and endoscopic approaches for neoplasia with non-exposure technique (CLEAN-NET) for gastric submucosal tumors: updated advantages and limitations. Ann Transl Med 2019;7(20):582. doi: 10.21037/atm.2019.09.19 risk of peritoneal dissemination. Br J Surg 2007;94:992-5.

29. Goto O, Takeuchi H, Kawakubo H, et al. First case of non-exposed endoscopic wall-inversion surgery with sentinel node basin dissection for early gastric cancer. Gastric Cancer 2015;18:434-9.

30. Goto O, Takeuchi H, Kitagawa Y, et al. Hybrid surgery for early gastric cancer. Transl Gastroenterol Hepatol 2016;1:26.

31. Takeuchi H, Goto O, Yahagi N, et al. Function-preserving gastrectomy based on the sentinel node concept in early gastric cancer. Gastric Cancer 2017;20:53-9.

32. Takeuchi M, Takeuchi H, Kawakubo H, et al. Update on the indications and results of sentinel node mapping in upper GI cancer. Clin Exp Metastasis 2018;35:455-61.

33. Hiramatsu Y, Takeuchi H, Goto O, et al. Minimally Invasive Function-Preserving Gastrectomy with Sentinel Node Biopsy for Early Gastric Cancer. Digestion 2019;99:14-20.

34. Kato M, Uraoka T, Isobe $Y$, et al. A case of gastric adenocarcinoma of fundic gland type resected by combination of laparoscopic and endoscopic approaches to neoplasia with non-exposure technique (CLEAN-NET). Clin J Gastroenterol 2015;8:393-9. 